\title{
Long-term outcomes of non-systematic device reimplantation following lead extraction in selected patients
}

\author{
WENCHENG ZHENG ${ }^{1-3^{*}}$, FENG ZE $^{1,2^{*}}$, DANDAN YANG ${ }^{1,2}$, DING LI $^{1,2}$, \\ $\mathrm{XU}_{\mathrm{ZHOU}}{ }^{1,2}$, CUIZHEN YUAN ${ }^{1,2}$ and XUEBIN LI ${ }^{1,2}$ \\ ${ }^{1}$ Department of Cardiac Electrophysiology, Peking University People's Hospital, Peking University; \\ ${ }^{2}$ Key Laboratory of Molecular Cardiovascular Science, Ministry of Education, Beijing 100044; \\ ${ }^{3}$ Fourth Department of Cardiovascular, Tangshan Gongren Hospital, Tangshan, Hebei 063000, P.R. China
}

Received March 29, 2021; Accepted August 3, 2021

DOI: $10.3892 / \mathrm{mi} .2021 .11$

\begin{abstract}
Following the removal of cardiovascular implantable electronic devices (CIEDs), reassessment of the need for a new device is vital. Some patients may have exhibited an improvement in rhythm or cardiac function and may thus no longer meet the guideline requirements for reimplantation. However, the long-term outcomes of non-systematic device reimplantation remain unknown. In the present study, it was hypothesized that the implantation of pacing systems in selected patients following lead extraction is safe. In order to confirm this hypothesis, a total of 854 patients (aged between 28 and 82 years) who underwent the removal of a CIED were enrolled in the present study and they were all reassessed to determine whether a new device following lead extraction was necessary. In order to determine which patients would undergo non-systematic device reimplantation, the standard guidelines, the criteria and the wishes of the patient were all taken into consideration. Patients remained device-free unless an adverse clinical event occurred that required reimplantation. The primary study endpoint was the rate of sudden death or reimplantation. Between January, 2014 and December, 2019, 854 consecutive patients underwent pacing system extraction, of whom 210 patients $(24.6 \%)$ underwent non-systematic device reimplantation following careful reassessment (the non-reimplantation group). Among the 210 patients, $162(77.1 \%)$ were fitted with pacemakers, $26(12.4 \%)$ underwent cardiac resynchronization therapy or cardiac resynchronization therapy-defibrillator and $22(10.5 \%)$ were implanted with
\end{abstract}

Correspondence to: Dr Feng Ze, Department of Cardiac Electrophysiology, Peking University People's Hospital, Peking University, 11 Xizhimen South Street, Xicheng, Beijing 100044, P.R. China

E-mail: zwczf984929@126.com

*Contributed equally

Key words: non-systematic device reimplantation, lead extraction, pacemaker a cardioverter-defibrillator. During a mean follow-up period of 40.4 months, 86 patients reached the primary endpoint of the study, including 54 out of 210 patients $(25.7 \%)$ who experienced an adverse clinical event that required reimplantation and 32 out of 210 patients (15.2\%) who experienced sudden death. Reimplantation of a new device was not required in $\sim 25 \%$ of the patients. On the whole, the present study demonstrates that following pacing system removal, non-systematic device reimplantation associated with close surveillance is safe for selected patients.

\section{Introduction}

The number of cardiovascular implantable electronic devices (CIEDs) has increased significantly over the past decade, and their use has improved the quality of life and survival rates among certain groups of patients (1). Accordingly, the need for the removal of CIEDs has also increased over the years (2-6). However, although the extraction techniques and tools have improved, the lead extraction procedure is still considered a challenging operation, and may even be life-threatening (7-10). The 2009 Heart Rhythm Society (HRS) Expert Consensus on Facilities, Training, Indications and Patient Management recommends that each patient should be carefully evaluated to determine whether there is a continued need for a new CIED (2). The 2017 updated HRS consensus statement further addresses the importance of the reassessment of the need for a new device (11). Reassessment of the need for a patient requiring a new CIED is imperative following the removal of an infected CIED. Some patients may have exhibited an interval improvement in rhythm or cardiac function and may thus no longer meet a guideline indication for a permanent pacemaker (PM), implantable cardioverter-defibrillator (ICD), or cardiac resynchronization therapy (CRT), or a patient may not wish to receive a new device due to financial difficulties or other reasons. The optimal timing of device replacement is unknown (12). To the best of our knowledge, to date, there are no prospective trial data on the timing of new device replacement and the risk of relapsing infection. A new implantation can reasonably be postponed until blood cultures are negative for $72 \mathrm{~h}$, which manifested that the bacteria were destroyed and the risks of reinfection for reimplantation were very 
low, although implantation should be delayed if the patient has another undrained source of infection, such as a psoas abscess. Previous pacing indications may have changed, and some arrhythmia may have resolved; thus, a reassessment of the need for a new device is imperative following the removal of a CIED. In order to provide optimal guidance for patients who required the removal of a CIED, it is important to also determine the safety and feasibility of reimplantation. The present study was performed to examine the long-term clinical outcomes and risks of non-systematic reimplantation following CIED removal.

\section{Patients and methods}

Patients. A single-center cohort of consecutive patients between January, 2014 and December, 2019 was studied. Retrospective analysis was performed using patient information from 2014 to 2018 and prospective analysis was performed using patient information from 2018 to 2019. The present study was approved by the Ethics Committee of Peking University People's Hospital (Beijing, China). All patients enrolled provided written informed consent for their participation in the study. Patients who were referred to the Cardiovascular Center of The Peking University People's Hospital (Beijing, China) for the removal of a CIED were enrolled in the study, in whom the device and lead were completely removed. All patients met the guideline criteria for lead extraction (11), including infectious and non-infectious reasons. The lead was extracted using laser sheaths, percutaneous mechanical techniques with dedicated instrumentation and needle snares, as appropriate. Patients who did not survive during the extraction procedures or did not wish to be enrolled were excluded from the study.

Electrode extraction. The lead was removed under general anesthesia and transesophageal ultrasound monitoring in high-risk patients, and the remaining patients were subjected to local anesthesia. Patients who were PM-dependent were implanted with a right ventricular (RV) pacing lead through the left femoral vein. Bilateral elbow venography was performed prior to extraction. The lead devices were removed using a manual or locking stylet traction (Cook Medical) in the case where were implanted 12 months prior to enrolment. The femoral vein approach was used with an evolution mechanical sheath (Cook Medical) and/or a laser sheath (CVX-300; Cook Medical). These methods were applied alone or in combination in patients with an implantation duration of $>12$ months. Endocardial active fixation ventricular electrodes were implanted in the patients who were PM-dependent as a transition to permanent PM reimplantation. In order to evaluate whether the patient required temporary pacing following electrode extraction, the PMs were programmed to the VVI mode at a back-up rate of $40 \mathrm{bpm}$ and monitored for $48 \mathrm{~h}$ prior to the procedure. Following the removal of the CIED, all patients were evaluated by the lead extraction team, including infectious disease experts, cardiologists and cardiac surgeons, who performed the extraction procedures. Each patient was carefully evaluated to determine whether there was a continued need for a new CIED. If the patients did not meet the non-reimplantation criteria, i.e., they were not PM-independent, had a high-degree atrioventricular block (AVB) and symptomatic bradycardia, the reimplantation was performed according to the guidelines (11). If the patients met the non-reimplantation criteria, such as: Patients had no history of syncope, they were PM-independent, the spontaneous ventricular rate was $>50 \mathrm{bpm}$, they had no high-degree AVB (Mobitz II or third-degree AVB), no chronic fascicular block, no symptomatic low rate $<50 \mathrm{bpm}$ and/or a sinus pause $>3 \mathrm{sec}$ during monitoring, or if the patients do not wish to undergo PM reimplantation (Table I), all the patients were placed under continuous cardiac monitoring for 7 days before being discharged from the Peking University People's Hospital. They remained device-free unless an adverse clinical event occurred that required reimplantation. All the patients were carefully followed-up every month for at least 3 years.

Criteria for non-reimplantation. The decision for the non-reimplantation of a pacing system was based on the guidelines of the 2017 HRS expert consensus statement on cardiovascular implantable electronic device lead management and extraction (11), as well as other separate factors, such as economic issues or patient wishes. The situation was discussed with the patient and their family members in order to reach a final decision (13-17). Following the removal of the CIED, the current clinical status, prognosis and wishes of the patients were reassessed. The criteria for non-reimplantation are listed in Table I.

Endpoint and follow-up. The primary study endpoint was the incidence of sudden death, and the rate of cardiac device reimplantation. All patients enrolled provided written informed consent for their participation in the study, and the present study was approved by the Ethics Committee of Peking University. The patients were then followed-up once a month at Peking University People's Hospital; three doctors at the center were responsible for the follow-up. An electrocardiogram (ECG/EKG), echocardiography and a 24-h Holter monitor were performed for the patients, after which a detailed evaluation would be made for each patient. For some patients who lived in remote areas, the survival and functional status were determined over the telephone. This evaluation was based on reported symptoms, a clinical examination and 24-h Holter monitoring findings (in the case that the patients could visit a local hospital and then forward the results).

Statistical analysis. Categorical variables are expressed as percentages and continuous variables are expressed as the mean \pm standard deviation. Non-parametric variables are expressed as the median and interquartile range. Continuous variables were compared between groups using the Student's t-test and Mann-Whitney U test, and categorical variables were compared using the $\chi^{2}$ test and Fisher's exact test. Survival analyses were performed using Kaplan-Meier curves and the log-rank test. For differences between three groups, one-Way ANOVA was use followed by post hoc analysis using the Bonferroni method. Statistical analyses were performed using SPSS 16.0 software (SPSS, Inc.). $\mathrm{P}<0.05$ was considered to indicate a statistically significant difference. 
Table I. Required criteria for the non-reimplantation strategy used in the present study.

Criteria for non-reimplantation

Total cohort $(n=210)$

Criteria for non-reimplantation of pacemaker

$(n=162)$

Patient's wishes

No history of syncope

Pacemaker-independent, spontaneous ventricular rate was $>50 \mathrm{bpm}$

No high-degree of AVB (Mobitz II or third-degree AVB)

No chronic fascicular block

No symptomatic low rate $<50 \mathrm{bpm}$ and/or a sinus pause $>3 \mathrm{sec}$ during monitoring

Criteria for non-reimplantation of CRT

Patient's wishes

Pacemaker-independent

Patients who had a LVEF $\leq 35 \%$, sinus rhythm, a non-LBBB pattern with QRS duration

120 to $149 \mathrm{msec}$, and NYHA class III following lead extraction.

Patients in AF and have a QRS duration < 150 msec, LVEF > 35\%, NYHA Class II-III

following lead extraction. The patient did not exhibit AV nodal ablation

Criteria for non-reimplantation of ICD

$(n=22)$

Patient's wishes

Patient with primary prevention and no clinically relevant ventricular arrhythmia attack

since ICD implantation and a LVEF $\geq 35 \%$ following lead extraction

Patient with primary prevention and no clinically relevant ventricular arrhythmia attack

since ICD implantation and a life prognosis $<1$ year

Criteria for non-reimplantation of CRT-D

$(n=16)$

Patient's wishes

Fulfill the criteria for non-reimplantation of CRT-P and ICD

AVB, atrioventricular block; CRT, cardiac resynchronization therapy; LVEF, left ventricular ejection fraction; LBBB, left bundle-branch block; QRS, EKG Q wave/R wave/S wave; NYHA, New York Heart Association; ICD, implantable cardioverter-defibrillator; CRT-D, cardiac resynchronization therapy-defibrillator.

\section{Results}

Frequency of non-reimplantation. From a cohort of 854 patients undergoing device extraction at Peking University People's Hospital, 644 (75.4\%) successfully underwent the reimplantation of a new device on the contralateral side; however, $210(24.6 \%)$ patients were considered to not require a new device. The baseline characteristics of these 854 patients, including whether they underwent reimplantation or not, are presented in Table II. The mean age of the patients in the present study was $65.7 \pm 15.2$ years. The majority of the patients were male (71.4\%). Among the patients who underwent device removal, $716(83.8 \%)$ were implanted with a PM, 27 (3.2\%) were fitted with a CRT-pace (CRT-P), 47 (5.5\%) were fitted with a CRT-defibrillator (CRT-D) and 64 (7.5\%) were implanted with an ICD in Table III. The most common reason for lead extraction was infection (89.1\%; Table IV).

For patients in the non-systematic device reimplantation group (non- reimplantation group; Table II), the median time of implantation was 60 months (25-75\% range, 14.5-150 months) at the time of presentation. Compared with the reimplantation group, patients who underwent non-systematic device reimplantation had a higher infection rate (94.3 vs. $87.4 \%$; $\mathrm{P}<0.001$; Table IV), a higher prevalence of atrial fibrillation
(AF) with a long RR interval (61.0 vs. 43.2\%; $\mathrm{P}<0.001)$ and a lower prevalence of AVB (12.4 vs. 39.3\%; P<0.001; Table II). During the procedure of lead extraction, femoral snare was the most widely used (54.2\%; Table IV). No significant differences in left ventricular function, comorbidities or the type of device were observed between the implantation and non-reimplantation groups.

Non-infectious variables in the non-reimplantation group included lead malfunction, thrombosis, chronic pain and MRI indications (if patients required an MRI examination, they may choose to no undergo reimplanation). Of the 4 patients who had superior vena cava syndrome and underwent lead extraction, only 1 patient was subjected to the reimplantation of a new device. A further 2 patients had chronic pain and received a new device following lead extraction. The reasons for the loss of reimplantation indications are presented in Table V.

Follow-up outcomes. As shown in Tables II and VI, 210 patients in the non-reimplantation group were followed-up for a mean duration of 40.4 months (25-75\%; range, 24.3-56.5 months). A total of 86 patients (40.9\%) reached the primary endpoint, including $32(15.2 \%)$ progressive deaths and $54(25.7 \%)$ reimplantation procedures. Of the 54 patients who were subjected to reimplantation, $35(16.7 \%)$ patients were subjected 
Table II. Baseline characteristics of the patients in the present study.

\begin{tabular}{|c|c|c|c|c|}
\hline Characteristic & $\begin{array}{l}\text { Non-reimplantation } \\
\qquad(\mathrm{n}=210)\end{array}$ & $\begin{array}{l}\text { Re-implantation } \\
(\mathrm{n}=644)\end{array}$ & $\begin{array}{l}\text { Total cohort } \\
\qquad(\mathrm{n}=854)\end{array}$ & P-value \\
\hline Age (years) & $66.6 \pm 13.1$ & $65.4 \pm 15.8$ & $65.7 \pm 15.2$ & 0.308 \\
\hline Male, n (\%) & $132(62.9)$ & $478(74.2)$ & $610(71.4)$ & 0.002 \\
\hline $\operatorname{LVEF}(\%)$ & $62.0 \pm 12.5$ & $61.9 \pm 12.2$ & $61.9 \pm 12.3$ & 0.912 \\
\hline Implant-time (months), median (IQR) & $60(14.5-150)$ & $60(23-120)$ & $60(24-120)$ & 0.909 \\
\hline $\mathrm{SND}, \mathrm{n}(\%)$ & $10(4.8)$ & $25(3.9)$ & $35(4.1)$ & 0.693 \\
\hline AVB, n (\%) & $26(12.4)$ & $253(39.3)$ & $279(32.7)$ & $<0.001$ \\
\hline Heart failure, n (\%) & $26(12.4)$ & $48(7.5)$ & $74(8.7)$ & 0.034 \\
\hline AF with long RR interval, n (\%) & $128(61.0)$ & $278(43.2)$ & $406(47.5)$ & $<0.001$ \\
\hline Ischemic cardiomyopathy, n (\%) & $20(9.5)$ & $37(5.7)$ & $57(6.7)$ & 0.057 \\
\hline Hypertrophic cardiomyopathy, n (\%) & $0(0)$ & $1(0.2)$ & $1(0.1)$ & 0.568 \\
\hline Inherited primary arrhythmia, $\mathrm{n}(\%)$ & $0(0)$ & $2(0.3)$ & $2(0.2)$ & 0.419 \\
\hline Hypertension, n (\%) & $94(44.8)$ & $268(41.6)$ & $362(42.4)$ & 0.423 \\
\hline Coronary artery disease, n (\%) & $33(15.7)$ & $125(19.4)$ & $158(18.5)$ & 0.231 \\
\hline Stroke, n $(\%)$ & $7(5.9)$ & $30(7.1)$ & $37(6.8)$ & 0.844 \\
\hline Valvular heart disease, n (\%) & $29(13.8)$ & $93(14.4)$ & $122(14.3)$ & 0.820 \\
\hline Diabetes mellitus, n (\%) & $36(17.1)$ & $144(22.4)$ & $180(21.1)$ & 0.107 \\
\hline Chronic renal failure, $\mathrm{n}(\%)$ & $10(4.8)$ & $41(6.4)$ & $51(6.0)$ & 0.394 \\
\hline
\end{tabular}

AVB, atrioventricular block; LVEF, left ventricular ejection fraction; SND, sinus node disease; AF, atrial fibrillation. Data are the mean \pm SEM; $\mathrm{P}<0.05$ was considered to indicate a statistically significant difference. Values represent the non-reimplantation vs. reimplantation groups only.

Table III. Characteristics of pacemaker device.

\begin{tabular}{lccc}
\hline & Non-reimplantation $(\mathrm{n}=210)$ & Re-implantation $(\mathrm{n}=644)$ & Total cohort $(\mathrm{n}=854)$ \\
\hline PM, $(\%)$ & $162(77.1)$ & $554(86.0)$ & $716(83.8)$ \\
CRT-P, n (\%) & $10(4.8)$ & $17(2.6)$ & $27(3.2)$ \\
CRT-D, n (\%) & $16(7.6)$ & $31(4.8)$ & $47(5.5)$ \\
ICD, n $(\%)$ & $22(10.5)$ & $42(6.5)$ & $64(7.5)$ \\
\hline
\end{tabular}

PM, pacemaker; CRT-P, cardiac resynchronization therapy-pace; CRT-D, cardiac resynchronization therapy-defibrillator; ICD, implantable cardioverter-defibrillator.

Table IV. Patients who underwent lead extraction and the associated parameters/tools.

\begin{tabular}{lccc}
\hline Lead extraction & Non-reimplantation $(\mathrm{n}=210)$ & Re-implantation $(\mathrm{n}=644)$ & Total cohort $(\mathrm{n}=854)$ \\
\hline Infection, $\mathrm{n}(\%)$ & $198(94.3)$ & $563(87.4)$ & $761(89.1)$ \\
Local infection, $\mathrm{n}(\%)$ & $171(86.4)$ & $515(91.5)$ & $686(90.1)$ \\
Systemic infection, $\mathrm{n}(\%)$ & $10(5.0)$ & $18(3.2)$ & $28(3.7)$ \\
Bacteremia, $\mathrm{n}(\%)$ & $17(8.6)$ & $30(5.3)$ & $47(6.2)$ \\
Femoral snare, $\mathrm{n}(\%)$ & $123(58.6)$ & $340(52.8)$ & $463(54.2)$ \\
Laser sheaths, $\mathrm{n}(\%)$ & $15(7.1)$ & $46(7.1)$ & $61(7.1)$ \\
Mechanical dilator sheaths, $\mathrm{n}(\%)$ & $4(1.9)$ & $15(2.3)$ & $19(2.2)$ \\
Surgical procedure, $\mathrm{n}(\%)$ & $2(1.0)$ & $1(0.2)$ & $3(0.4)$ \\
Locking stylets, $\mathrm{n}(\%)$ & $47(22.4)$ & $94(14.6)$ & $195(22.8)$ \\
Others ${ }^{\mathrm{a}}, \mathrm{n}(\%)$ & $19(9.0)$ & & $113(13.2)$ \\
\hline
\end{tabular}

${ }^{\mathrm{a} C}$ Combination of two or more tools. 
Table V. Reason for the loss of reimplantation indications.

Reason for loss of reimplantation

Total $(\mathrm{n}=210)$

Pacemaker
Pacemaker-independent and no long RR interval observed
Patients with AF accept radiofrequency ablation
Patients who passed away without bradycardia-related symptoms
ICD
No ventricular arrhythmia observed
CRT/CRT-D
Patients with AF and a QRS duration $<150$ msec, a LVEF $>35 \%$ and NYHA Class II-III
following lead extraction
Patients with sinus rhythm, LVEF $>35 \%$ and NYHA Class I-II following lead extraction

$(n=16)$

ICD, implantable cardioverter-defibrillator; CRT, cardiac resynchronization therapy; CRT-D, cardiac resynchronization therapy-defibrillator; LVEF, left ventricular ejection fraction; AF, atrial fibrillation; NYHA, New York Heart Association; QRS, EKG Q wave/R wave/S wave.

Table VI. Characteristics of the 210 patients in the nonreimplantation group.

\begin{tabular}{lc}
\hline Characteristics of non-reimplantation & Total $(\mathrm{n}=210)(\%)$ \\
\hline Patients who reached the endpoint & $86(40.9)$ \\
Progressive death & $32(15.2)$ \\
Reimplantation procedure & $54(25.7)$ \\
Reimplantation in the second year & $35(16.7)$ \\
Reimplantation in the third year & $10(4.8)$ \\
Reimplantation $>3$ years & $9(4.3)$ \\
Overall mortality rate & $32(15.2)$ \\
Cancer & $10(4.8)$ \\
Heart failure & $4(1.9)$ \\
Stroke & $8(3.8)$ \\
Peripheral artery disease & $3(1.4)$ \\
Renal failure & $7(3.3)$ \\
\hline
\end{tabular}

to reimplantation in the second year, $10(4.8 \%)$ in the third year and $9(4.3 \%)$ after $>3$ years. The median time of implantation was 60 months (25-75\% range, 14.5-150 months).

The overall mortality rate was $15.2 \%(n=32)$, and the reasons for this included cancer $(4.8 \% ; \mathrm{n}=10)$, heart failure $(1.9 \% ; n=4)$, stroke $(3.8 \% ; n=8)$, peripheral artery disease $(1.4 \% ; n=3)$ and renal failure $(3.3 \% ; n=7)$. All patients who did not survive had undergone non-systematic device reimplantation and none had suffered bradycardia-related symptoms prior to their death.

As shown in Tables II and VII, no significant differences were observed in age, sex and follow-up results between the different device type groups. Compared with the PM group, the patients previously implanted with CRT-P/D or ICD, had a lower left ventricular ejection fraction (LVEF; $\mathrm{P}<0.001$ ). No significant differences were observed in the reimplantation rate ( 26.5 vs. 11.5 vs. $36.4 \%, \mathrm{P}=0.565)$ and mortality (13.6 vs. 19.2 vs. $22.7 \%, \mathrm{P}=0.565$ ) between the PM, CRT-P/D and ICD groups, respectively.

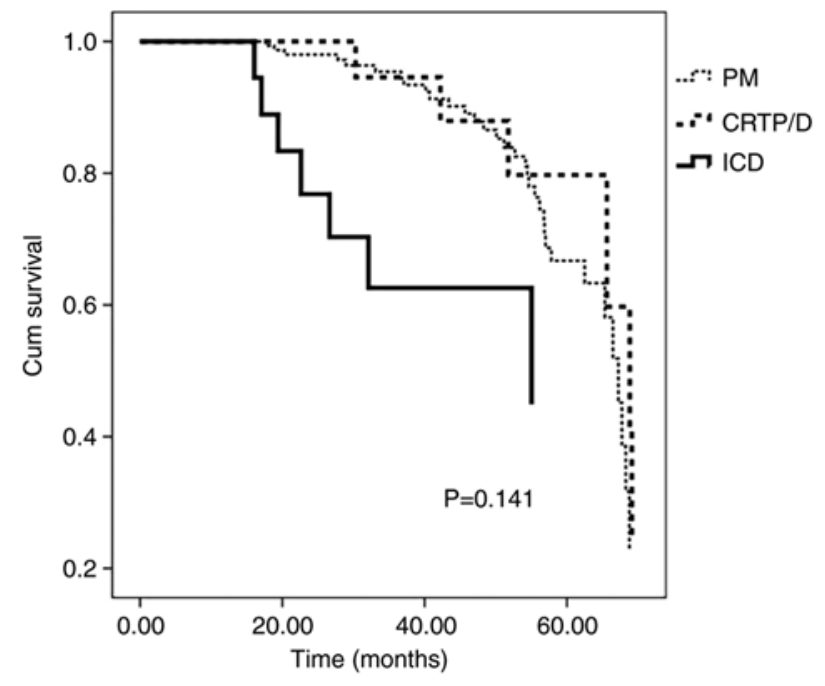

Figure 1. Survival analysis of non-reimplantation patients in different device groups. Distribution of endpoint events (progressive deaths and reimplantation) according to the follow-up time. Kaplan-Meier curves for endpoint events are presented for both groups. There were no significant differences between the three groups $(\mathrm{P}=0.141)$.

In the CRT-P/D group ( $\mathrm{n}=26$; Table VII), which included 10 patients implanted with CRT-P and 16 with CRT-D, 3 patients $(11.5 \%)$ underwent reimplantation. A total of 2 patients previously implanted with CRT-P underwent reimplantation and accepted the AV nodal ablation due to AF, and 1 patient previously fitted with CRT-D refused reimplantation following device removal; however, this patient did have to undergo reimplantation during the follow-up time due to the worsening of heart function. Due to primary prevention ICD at the initial implant, no clinically relevant ventricular arrhythmias were observed following the implantation of CRT-D; thus, the patient downgraded from the CRT-D to the CRT-P device during the follow-up time. In the CRT-P/D group $(n=26)$, 5 patients (19.2\%) died during the follow-up period; however, this was not due to cardiac reasons ( 3 were the result of a stroke and 2 were due to cancer). 
Table VII. Follow-up results of patients in the non-reimplantation group between different device groups.

\begin{tabular}{lcccc}
\hline Characteristic & $\begin{array}{c}\text { PM Group } \\
(\mathrm{n}=162)\end{array}$ & $\begin{array}{c}\text { CRT-P/D Group } \\
(\mathrm{n}=26)\end{array}$ & $\begin{array}{c}\text { ICD group } \\
(\mathrm{n}=22)\end{array}$ & $\begin{array}{c}\text { Total cohort } \\
(\mathrm{n}=210)\end{array}$ \\
\hline Age (years) & $67.7 \pm 12.6$ & $63.4 \pm 12.2$ & $62.4 \pm 16.4$ & $66.6 \pm 13.1$ \\
Male, $\mathrm{n}(\%)$ & $97(59.9)$ & $18(69.2)$ & $17(77.3)$ & $132(62.9)$ \\
LVEF (\%) & $65.1 \pm 10.0$ & $50.3 \pm 14.3^{\mathrm{a}, \mathrm{b}}$ & $52.3 \pm 14.6^{\mathrm{a}, \mathrm{b}}$ & $62.0 \pm 12.3$ \\
No symptom, $\mathrm{n}(\%)$ & $76(46.9)$ & $16(61.5)$ & $7(31.8)$ & $99(47.1)$ \\
Reimplantation, $\mathrm{n}(\%)$ & $43(26.5)$ & $3(11.5)$ & $8(36.4)$ & $54(25.7)$ \\
Poor wound healing, $\mathrm{n}(\%)$ & $2(1.2)$ & 0 & 0 & $2(1.0)$ \\
Mortality, $\mathrm{n}(\%)$ & $22(13.6)$ & $5(19.2)$ & $5(22.7)$ & $32(15.2)$ \\
Ablation or surgical procedure, $\mathrm{n}(\%)$ & $5(3.1)$ & 0 & $1(4.5)$ & $6(2.9)$ \\
Others & $14(8.6)$ & $2(7.8)$ & $1(4.5)$ & $17(8.1)$ \\
\hline
\end{tabular}

${ }^{\mathrm{a}} \mathrm{P}<0.05$ vs. PM group; ${ }^{\mathrm{b}} \mathrm{P}>0.05$ vs. CRT-P/D group. PM, pacemaker; CRT-P, cardiac resynchronization therapy-pace; CRT-D, cardiac resynchronization therapy-defibrillator.

The patients in the non-reimplantation group and according to the different devices were as follows: In the PM group, 162 patients did not accept reimplantation, and in the CRT-P, CRT-D and ICD groups, the number of non-reimplantation patients were 10, 16 and 22, respectively (Table III).

In the ICD group $(n=22), 8$ patients $(36.4 \%)$ underwent reimplantation procedures during the follow-up period (Table VII). Although no clinically relevant ventricular tachycardia was observed following the implantation of the ICD, and the LVEF was $\geq 35 \%$ following lead extraction, 4 patients $(18.2 \%)$ with ICD primary prevention also underwent reimplantation due to the occurrence of ventricular arrhythmia. A total of 4 patients belonged to the ICD secondary prevention group; however, they refused reimplantation following device removal. Among the 22 ICD patients, a heart transplant procedure was performed for 1 (4.5\%), whereas 5 (22.7\%) passed away during the follow-up period; however, this was not to ventricular arrhythmia ( 3 died of heart failure, 1 of cancer and 1 of renal failure).

As shown in Table VII, the use of different device types was not significantly associated with increased endpoint events for age, sex and ejection fraction. In addition, as shown in Fig. 1, the survival analysis of the non-reimplantation patients in the different device groups did not reveal significant differences between groups $(\mathrm{P}=0.141)$. However, there was a trend toward worse outcomes in the ICD group.

\section{Discussion}

The timing of the reimplantation of a new device system following the extraction of an infected device remains a subject of debate and is influenced by the causative agent and clinical presentation. Antimicrobial therapy according to the 2017 HRS expert consensus recommendations (11) was used for the management of the suspected electronic device-associated infection. The antimicrobial therapy used for endocarditis has a minimum duration of 4-6 weeks, whereas for bacteremia, it has a lower duration (at least 2 weeks). A new implantation may be reasonably postponed until blood cultures are negative for
$72 \mathrm{~h}$ in patients with bacteremia and endocarditis. In subjects with pocket site infection, the reimplantation procedure was performed with a treatment duration of 48-72 $\mathrm{h}$ following device removal. Reassessment of the need for a new device is imperative following the removal of a CIED. To the best of our knowledge, this is the first study to assess the long-term outcomes of non-systematic device reimplantation following the removal of a CIED. It is necessary to evaluate whether patients require new device reimplantation or not following the removal of a device system. The criteria mentioned in the present study may be useful to reassess the need for reimplantation. A total of 854 patients were analyzed in the present study, $210(24.6 \%)$ of whom underwent non-systematic device reimplantation following careful reassessment (Table II). During a mean follow-up of 40.4 months, out of the 210 patients, 86 $(40.9 \%)$ reached the primary endpoint, which included 32 $(15.2 \%)$ patients who passed away and $54(25.7 \%)$ who accepted reimplantation procedures. All the patients that did not survive had undergone non-systematic device reimplantation and none had suffered bradycardia-related symptoms prior to passing away. No significant differences were observed in the endpoint events between the different device type groups (Table VII). These findings suggested that following the removal of pacing systems, non-systematic device reimplantation associated with close surveillance was safe in selected patients.

In the present study, the most common reason for device removal was infection (Table III). The need for the implantation of a new device system should be carefully assessed in all patients who undergo the removal of a CIED (18). Previous pacing indications, such as hypertrophic cardiomyopathy pacing to relieve the outflow obstruction or vasovagal syncope syndrome may no longer be strong indications, as post-operative conduction disorders may have improved. It has been reported that $13-52 \%$ of patients do not require pacing devices after the infected system has been removed (19-22). In the present study, the reimplantation of a new device was not required in $\sim 25 \%$ of patients.

A common reason for non-systematic device reimplantation is the development of persistent AF from sinus rhythm. 
In the present study, some patients underwent ablation or surgical procedures. It is reasonable to offer AF ablation as an alternative to pacemaker implantation in patients with tachycardia-bradycardia syndrome. Class IIa guidelines recommend that patients with tachycardia-bradycardia syndrome should undergo ablation procedures (23). Therefore, in the present study, while some patients underwent CIED implantation due to tachycardia-bradycardia syndrome, AF ablation may be an alternative option following device removal. Superior vena cava occlusion may have occurred following lead extraction, which renders the reimplantation of a new device difficult; thus, the implantation of leadless PMs may be an option. In the present study, 7 patients did not accept the reimplantation immediately, in the follow-up time they underwent ablation procedures (5 patients) or leadless PM implantation (2 patients).

Patients who undergo CRT are usually independent; thus, there is sufficient time to reassess the situation of the patient following lead extraction. The reimplantation of a biventricular device is often performed from the right side, where operators have less experience; moreover, from the right subclavian vein to the coronary sinus, there are two physical curves; the other major difficulty in the reimplantation of left ventricular lead (LVL) following removal comes from occlusion or stenosis of the original implanted branches, which all result in difficulties during the implantation of the LVL (18). Rickard et al (18) performed a retrospective study that included 151 patients who underwent the extraction of a biventricular pacing device due to infection. They found that patients with biventricular device infection who were deemed to be cured and underwent successful reimplantation of a biventricular device exhibited a trend towards improved outcomes compared with patients deemed cured who did not undergo successful CRT reimplantation (18). The beneficial effects of biventricular pacing in the reimplantation group may also have played an important role. It is hoped that the benefits of biventricular pacing will be preserved, which is a new system that should be reimplanted immediately following lead extraction; however, the optimal time for undergoing biventricular device reimplantation remains to be elucidated. In the CRT-P/D group, $11.5 \%$ of patients underwent reimplantation and 1 patient downgraded from the CRT-D to the CRT-P device during the follow-up period. The present study suggested that non-systematic device reimplantation in biventricular pacing patients was safe, $>50 \%$ of the patients remained device-free and no patients appeared to exhibit worse outcomes. No significant differences were observed in the mortality rate of the patients reimplanted with non-systematic devices, which may be due to the higher LVEF observed in this group that may play an important role in the success of this treatment.

Previous research (24) has reported that there are no factors that can predict the decreased need for ICD therapy. Hence, ICD replacement appears to remain necessary in patients without prior ICD interventions. A previous study found that the majority of patients that underwent ICD primary prevention do not experience a ventricular accident during the first battery service-life (25). However, a substantial number of these patients do receive appropriate ICD therapy following replacement. During the follow-up period following replacement, the 3 -year cumulative incidence of appropriate therapy in response to ventricular tachycardia or ventricular fibrillation has been found to be $14 \%$ (25). In the present study, although no clinically relevant ventricular arrhythmias were observed following ICD implantation, and LVEF was $\geq 35 \%$ following lead extraction, 4 (18.2\%) patients with primary prevention ICD underwent reimplantation due to the occurrence of ventricular arrhythmia.

The present study found that patients with ICD who did not undergo reimplantation exhibited a trend toward worse outcomes compared with patients deemed as cured and who underwent successful reimplantation with PM and biventricular devices. The reason is most likely that the condition of the non-reimplanted patients was more severe at baseline (they were noted to have a lower LVEF).

A previous 12-month study found that it was safe to not reimplant pacemakers following the extraction of infected systems in low-risk patients, allowing the administration of antimicrobials in a device-free state (26), which was consistent with the findings of the present study. These patients should be followed-up further, and an implantable electrocardiography loop recorder may be helpful in the decision-making process following the removal of the devices.

The present study has several limitations which should be noted. First, the collection of data was partly retrospective and some patients resided in remote areas; thus, survival and functional statuses had to be confirmed via telephone. Details of the patients would need to be evaluated in-detail in future studies. Second, detailed follow-up information concerning patients undergoing reimplantation was not obtained, such as body mass index (BMI), New York Heart Association (NYHA) class (I, II, III and IV), ejection traction (\%) and hemoglobin A1c (HbA1c) (\%). Third, the efficiency and safety of the criteria at the Cardiac Electrophysiology Center, Peking University People's Hospital, should be tested in further studies. Moreover, the present study also has certain other limitations. The present study did calculate the mortality of patients following systematic reimplantation as part of the patients resided in remote areas and the follow-up was inconvenient. Thus, no information was obtained regarding the difference in mortality between the systematic reimplantation group and the non-reimplantation group. If the device was very new and the battery level was sufficient, perhaps the primary device could be reimplanted after being sterilized. If the device was very old and the battery level was close to the elective replacement indicator (ERI) level, it is suggested that patients are reimplanted with a new device. In addition, the present study did not perform a follow-up analysis of the long-term mortality of the infectious vs. non-infectious group, depending on reimplantation. Thus, further studies are required to focus on this matter.

In conclusion, as demonstrated in the present study, the reimplantation of a new device was not required in $25 \%$ of patients. Following the removal of pacing systems, non-systematic device reimplantation associated with close surveillance was found to be safe in selected patients.

\section{Acknowledgements}

Not applicable. 


\section{Funding}

No funding was received.

\section{Availability of data and materials}

All data generated or analyzed during the current study are included in this published article.

\section{Authors' contributions}

WZ and FZ performed the study and wrote the manuscript. WZ, FZ and DY contributed to data analysis and interpretation. WZ, FZ, DY, DL, XZ, CY and XL contributed to the whole process of the study (including conception and design, writing, literature search). WZ and FZ confirm the authenticity of the raw data.

\section{Ethics approval and consent to participate}

All experiments were conducted with the approval of the Ethics Committee of Peking University People's Hospital (Beijing, China). All patients provided informed consent after being informed in detail of the potential complications of the procedures performed in the present study.

\section{Patient consent for publication}

Not applicable.

\section{Competing interests}

The authors declare that they have no competing interests.

\section{References}

1. Matchett M, Sears SF, Hazelton G, Kirian K, Wilson E and Nekkanti R: The implantable cardioverter defibrillator: Its history, current psychological impact and future. Expert Rev Med Devices 6: 43-50, 2009.

2. Wilkoff BL, Love CJ, Byrd CL, Bongiorni MG, Carrillo RG, Crossley GH III, Epstein LM, Friedman RA, Kennergren CE, Mitkowski P, et al: Transvenous lead extraction: Heart rhythm Society expert consensus on facilities, training, indications, and patient management: This document was endorsed by the American heart association (AHA). Heart Rhythm 6: 1085-1104, 2009.

3. Sohal M, Williams SE, Arujuna A, Chen Z, Bostock J, Gill JS and Rinaldi CA: The current practice and perception of cardiac implantable electronic device transvenous lead extraction in the UK. Europace 15: 865-870, 2013.

4. Voigt A, Shalaby A and Saba S: Continued rise in rates of cardiovascular implantable electronic device infections in the United States: Temporal trends and causative insights. Pacing Clin Electrophysiol 33: 414-419, 2010.

5. Carrasco F, Anguita M, Ruiz M, Castillo JC, Delgado M, Mesa D, Romo E, Pan M and de Lezo JS: Clinical features and changes in epidemiology of infective endocarditis on pacemaker devices over a 27 -year period (1987-2013). Europace 18 : 836-841, 2016

6. Aljabri K, Garlitski A, Weinstock J and Madias C: Management of device infections. Card Electrophysiol Clin 10: 153-162, 2018.

7. Bashir J and Carrillo RG: Cardiac and vascular injuries sustained during transvenous lead extraction. Card Electrophysiol Clin 10: 651-657, 2018

8. Maytin M and Epstein LM: The challenges of transvenous lead extraction. Heart 97: 425-434, 2011
9. Hauser RG, Katsiyiannis WT, Gornick CC, Almquist AK and Kallinen LM: Deaths and cardiovascular injuries due to device-assisted implantable cardioverter-defibrillator and pacemaker lead extraction. Europace 12: 395-401, 2010.

10. Nof E, Bongiorni MG, Auricchio A, Butter C, Dagres N, Deharo JC, Rinaldi CA, Maggioni AP, Kutarski A, Kennergren C, et al: Comparison of outcomes in infected cardiovascular implantable electronic devices between complete partial, and failed lead removal: An ESC-EHRA-EORP ELECTRa (European Lead Extraction ConTrolled) registry. Europace 21: 1876-1889, 2019.

11. Kusumoto FM, Schoenfeld MH, Wilkoff BL, Berul CI, Birgersdotter-Green UM, Carrillo R, Cha YM, Clancy J, Deharo JC, Ellenbogen KA, et al: 2017 HRS expert consensus statement on cardiovascular implantable electronic device lead management and extraction. Heart Rhythm 14: e503-e551, 2017.

12. Chew D, Somayaji R, Conly J, Exner D and Rennert-May E: Timing of device reimplantation and reinfection rates following cardiac implantable electronic device infection: A systematic review and meta-analysis. BMJ Open 9: e029537, 2019.

13. Brignole M, Auricchio A, Baron-Esquivias G, Bordachar P, Boriani G, Breithardt OA, Cleland J, Deharo JC, Delgado V, ElliottPM, et al: 2013 ESC Guidelines on cardiac pacing and cardiac resynchronization therapy: The task force on cardiac pacing and resynchronization therapy of the European society of cardiology (ESC). Developed in collaboration with the European heart rhythm association (EHRA). Eur Heart J 34: 2281-2329, 2013.

14. Priori SG, Blomström-Lundqvist C, Mazzanti A, Blom N, Borggrefe M, Camm J, Elliott PM, Fitzsimons D, Hatala R, Hindricks G, et al: 2015 ESC Guidelines for the management of patients with ventricular arrhythmias and the prevention of sudden cardiac death: The task force for the management of patients with ventricular arrhythmias and the prevention of sudden cardiac death of the European society of cardiology (ESC)endorsed by: Association for European paediatric and congenital cardiology (AEPC). Europace 17: 1601-1687, 2015.

15. Ponikowski P, Voors AA, Anker SD, Bueno H, Cleland JG, Coats AJ, Falk V, González-Juanatey JR, Harjola VP, Jankowska EA, et al: 2016 ESC guidelines for the diagnosis and treatment of acute and chronic heart failure: The task force for the diagnosis and treatment of acute and chronic heart failure of the European society of cardiology (ESC)developed with the special contribution of the heart failure association (HFA) of the ESC. Eur Heart J 37: 2129-2200, 2016.

16. Shen WK, Sheldon RS, Benditt DG, Cohen MI, Forman DE, Goldberger ZD, Grubb BP, Hamdan MH, Krahn AD, Link MS, et al: 2017 ACC/AHA/HRS guideline for the evaluation and management of patients with syncope: A report of the American college of cardiology/American heart association task force on clinical practice guidelines and the heart rhythm society. J Am Coll Cardiol 70: e39-e110, 2017.

17. Kusumoto FM, Schoenfeld MH, Barrett C, Edgerton JR, Ellenbogen KA, Gold MR, Goldschlager NF, Hamilton RM, Joglar JA, Kim RJ, et al: 2018 ACC/AHA/HRS guideline on the evaluation and management of patients with bradycardia and cardiac conduction delay: A report of the american college of cardiology/American heart association task force on clinical practice guidelines and the heart rhythm society. Circulation 140: e382-e482, 2019.

18. Rickard J, Tarakji K, Cronin E, Brunner MP, Jackson G, Baranowski B, Borek PP, Martin DO, Wazni O and Wilkoff BL: Cardiac venous left ventricular lead removal and reimplantation following device infection: A large singal-center experience. J Cardiovasc Electrophysiol 23: 1213-1216, 2012.

19. Cacoub $P$, Leprince $P$, Nataf $P$, Hausfater $P$, Dorent $R$, Wechsler $B$, Bors V, Pavie A, Piette JC and Gandjbakhch I: Pacemaker infective endocarditis. Am J Cardiol 82: 480-484, 1998.

20. Lewis AB, Hayes DL, Holmes DR Jr, Vlietstra RE, Pluth JR and Osborn MJ: Update on infections involving permanent pacemakers: Characterization and management. J Thorac Cardiovasc Surg 89: 758-763, 1985.

21. Böhm Á, Bányai F, Préda I and Zámolyi K: The treatment of septicemia in pacemaker patients. Pacing Clin Electrophysiol 19: 1105-1111, 1996.

22. Chua JD, Wilkoff BL, Lee I, Juratli N, Longworth DL and Gordon SM: Diagnosis and management of infections involving implantable electrophysiologic cardiac devices. Ann Intern Med 133: 604-608, 2000. 
23. Calkins H, Hindricks G, Cappato R, Kim YH, Saad EB, Aguinaga L, Akar JG, Badhwar V, Brugada J, Camm J, et al: 2017 HRS/EHRA/ECAS/APHRS/SOLAECE expert consensus statement on catheter and surgical ablation of atrial fibrillation. Europace 20: e1-e160, 2018.

24. Erkapic D, Sperzel J, Stiller S, Meltendorf U, Mermi J, Wegscheider K and Hügl B; INSURE Investigators: Long-term benefit of implantable cardioverter/defibrillator therapy after elective device replacement: Results of the incidence free survival after ICD replacement (INSURE) trial-a prospective multicentre study. Eur Heart J 34: 130-137, 2013.

25. Van Welsenes GH, Rees JB, Thijssen J, Trines SA, Erven LV, Schalij MJ and Borleffs CJW: Primary prevention implantable cardioverter defibrillator recipients: The need for defibrillator back-up after an event-free first battery service-life. J Cardiovasc Electrophysiol 22: 1346-1350, 2011.
26. Marijon E, Guillebon MD, Bordachar P, Jacob S, Vahdat O, Sidobre L, Deplagne A, Combes N, Albenque JP, Clementy J, et al: Safety of deferring the reimplantation of pacing systems after their removal for innfectious complications in selected patients: A 1-year follow-up study. J Cardiovasc Electrophysiol 21: $540-544,2010$

(i) (9) This work is licensed under a Creative Commons Attribution-NonCommercial-NoDerivatives 4.0 International (CC BY-NC-ND 4.0) License. 